\title{
Mobile Technologies for Preservation of Indigenous Knowledge in Rural Communities
}

\author{
Heike Winschiers-Theophilus \\ School of Information Technology \\ Polytechnic of Namibia \\ Windhoek, Namibia \\ heikewinschiers@gmail.com
}

\author{
Kasper Rodil \\ Department of Architecture, Design and Media Technology \\ Alborg University \\ Aalborg, Denmark \\ kr@create.aau.dk
}

\author{
Tariq Zaman \& Alvin Yeo \\ Institute of Social Informatics \& Technological Innovations \\ University Malaysia Sarawak \\ Kuching, Malaysia \\ zamantariq@gmail.com, alvin@isiti.unimas.my
}

\author{
Kasper Jensen \\ Mobile Future Lab \\ Namibian Business Innovation Centre \\ Windhoek, Namibia \\ kasper.loevborg@gmail.com
}

\begin{abstract}
In this paper we explore the opportunities of mobile technologies in three of our own development endeavors with rural communities, promoting the preservation of indigenous knowledge. We reflect upon and recognize the fact that the representation of indigenous knowledge will be transformed within the digitalization process under the limitations and capabilities of the tools. We believe that a continuation of local appropriation and co-design of tools will lead to an integrated, intuitive and non-intrusive indigenous knowledge preservation process within the local communities.
\end{abstract}

Keywords-Indigenous Knowledge, mobile technology, smart phones, rural communities

\section{DIGITAL PRESERVATION OF INDIGENOUS KNOWLEDGE}

Technologies to support storage, processing and distribution of knowledge have evolved over decades within a pre-dominantly written knowledge paradigm. Thus, a variety of well-established tools for text based knowledge assimilation and dissemination are available, such as e-readers with annotation facilities, meta-data and semantic search facilities, text-based knowledge sharing platforms and distributed databases. Following this pattern a number of Indigenous Knowledge Databases were created and populated following an entity-relational abstraction model as supported by mainstream technologies. However, more than a decade ago, researchers have already alerted technology designers of the misrepresentation of non-text-based knowledge systems. [1] for example exposed the discrepancies between the Maori knowledge organization and the commonly used library metaphor. Followed by many attempts of modeling 'other' cultures and knowledge systems, yet neither theoretical nor practical outcomes have influenced mainstream technology design. This becomes even more problematic if the purpose of the technology is to support the preservation of Indigenous Knowledge (IK) itself.

Thus a major concern has been the lack of involvement of indigenous communities in the actual design of technologies to shape IK preservation and curation in accordance with their own representational choices. In many instances IK is collected by outsiders through "ethnographical" means such as observations, interviews, video and audio recordings and then equally curated by outsiders for outsiders. Interestingly, only few research or development initiatives have focused on the indigenous communities being the end-users of technologies supporting their own knowledge collection, curation and dissemination.

In this paper we explore the opportunities of mobile technologies in three of our own development endeavors with rural indigenous communities. We reflect upon and recognize the fact that the representation of indigenous knowledge will be transformed within the digitalization process under the limitations and capabilities of the tools. Yet we consider the involvement of local communities as an essential contribution to the long term technology design and adoption.

\section{COLLABORATION BACKGROUND}

The authors, one based in Borneo and three engaged in projects in Namibia have been collaborating on a conceptual level as well as on a site exchange visit basis. Although the development contexts seem in many ways different, e.g. one site being in the Rainforest while the other in the Kalahari Desert, they have a number of fundamental similarities allowing for transferability of best practices and design 